\title{
RASSEGNA
}

\section{L'irisina: un ormone con benefici multiorgano}

\author{
Giulia Le Grazie $^{1}$ - Nicola Marrano ${ }^{1}$ - Annalisa Natalicchio ${ }^{1}$. Francesco Giorgino ${ }^{1}$
}

Accettato: 24 gennaio 2022 / Pubblicato online: 3 marzo 2022

(c) The Author(s) 2022, corrected publication 2022

\section{Sommario}

L'irisina è una miochina secreta dal muscolo scheletrico in seguito ad attività fisica, in grado di regolare l'omeostasi glucidica ed energetica, agendo su numerosi tessuti e intervenendo su diversi pathways metabolici. Un'alterazione dei livelli sierici di irisina potrebbe promuovere l'insorgenza di patologie metaboliche, tra cui il diabete mellito di tipo 2. Numerosi studi su modelli animali di diabete e/o obesità hanno dimostrato che la somministrazione di irisina esogena è in grado di esercitare effetti antidiabetici e antiobesità.

Parole chiave Irisina $\cdot$ Diabete $\cdot$ Obesità $\cdot$ Metabolismo

\section{Introduzione}

L'irisina è un ormone di 112 aminoacidi ( $\sim 12 \mathrm{KDa})$ risultante dal clivaggio della porzione extracellulare del dominio N-terminale della proteina di membrana fibronectin type III domain contaning protein 5 (FNDC5) [1]. L'irisina è stata descritta per la prima volta nel 2012 da Boström e collaboratori [1] come una miochina secreta principalmente dal muscolo scheletrico in seguito ad attività fisica e in grado di favorire i processi di browning del tessuto adiposo bianco e di termogenesi. Numerosi studi hanno esplorato le proprietà pleiotropiche dell'irisina, dimostrando il suo ruolo fondamentale nella regolazione del metabolismo energetico, agendo su diversi tessuti e intervenendo in numerose vie biochimiche.

I livelli circolanti di irisina sono ridotti nei pazienti affetti da diabete mellito di tipo 2 (DMT2) [2]; al contrario, essi sono più elevati in altri stati dismetabolici caratterizzati da insulino-resistenza, come l'obesità [3], la sindrome metabolica [4, 5] e la sindrome dell'ovaio policistico [6], probabilmente riflettendo una condizione di resistenza all'irisina o un aumento compensatorio dovuto alle iniziali anomalie metaboliche che sono caratteristiche di questi pazienti [3-5].

Proposto da L. Laviola.

$凶$ A. Natalicchio

annalisa.natalicchio@uniba.it

1 Dipartimento dell'Emergenza e dei Trapianti di Organi, Sezione di Medicina Interna, Endocrinologia, Andrologia e Malattie Metaboliche, Università degli Studi di Bari Aldo Moro, Bari, Italia
A sostegno di quest'ultimo concetto, alcuni studi hanno riportato l'esistenza di correlazioni positive tra i livelli sierici di irisina e alcuni marker di adiposità e di disturbi dell'omeostasi del glucosio e dei lipidi [7]. Questo suggerisce la possibilità che l'alterazione dei livelli sierici di irisina possa contribuire ad alterare l'omeostasi metabolica ed energetica e promuovere l'insorgenza di patologie metaboliche, tra cui il DMT2. Infatti, numerosi studi interventistici su modelli animali di diabete e/o obesità hanno dimostrato che la somministrazione di irisina esogena è in grado di ripristinare l'omeostasi glucidica e lipidica, esercitando così effetti antidiabetici e antiobesità [7].

\section{Effetti pleiotropici dell'irisina}

I molteplici e sistemici effetti benefici dell'irisina a livello del metabolismo energetico e glucidico riflettono la sua capacità di agire sui principali organi e tessuti coinvolti nella regolazione dell'omeostasi metabolica.

Tra le azioni di maggiore importanza, l'irisina è in grado di promuovere la sintesi dell'insulina e la secrezione insulinica glucosio-stimolata, ridurre l'apoptosi delle beta-cellule pancreatiche in risposta a diversi stimoli dannosi e favorire la proliferazione beta-cellulare [7-9]. Questi dati sono stati dimostrati in linee cellulari di beta-cellule murine e umane, in isole pancreatiche murine e umane, e in vivo in topi sani. Complessivamente, l'irisina è in grado di favorire il mantenimento e il ripristino della normale massa funzionale beta-cellulare, essenziale per un'adeguata omeostasi glucidica (Fig. 1). 
Fig. 1 Effetti dell'irisina a livello delle beta-cellule pancreatiche (modificata [7])

Fig. 2 Effetti pleiotropici dell'irisina (modificata da [7])

ISOLE PANCREATICHE
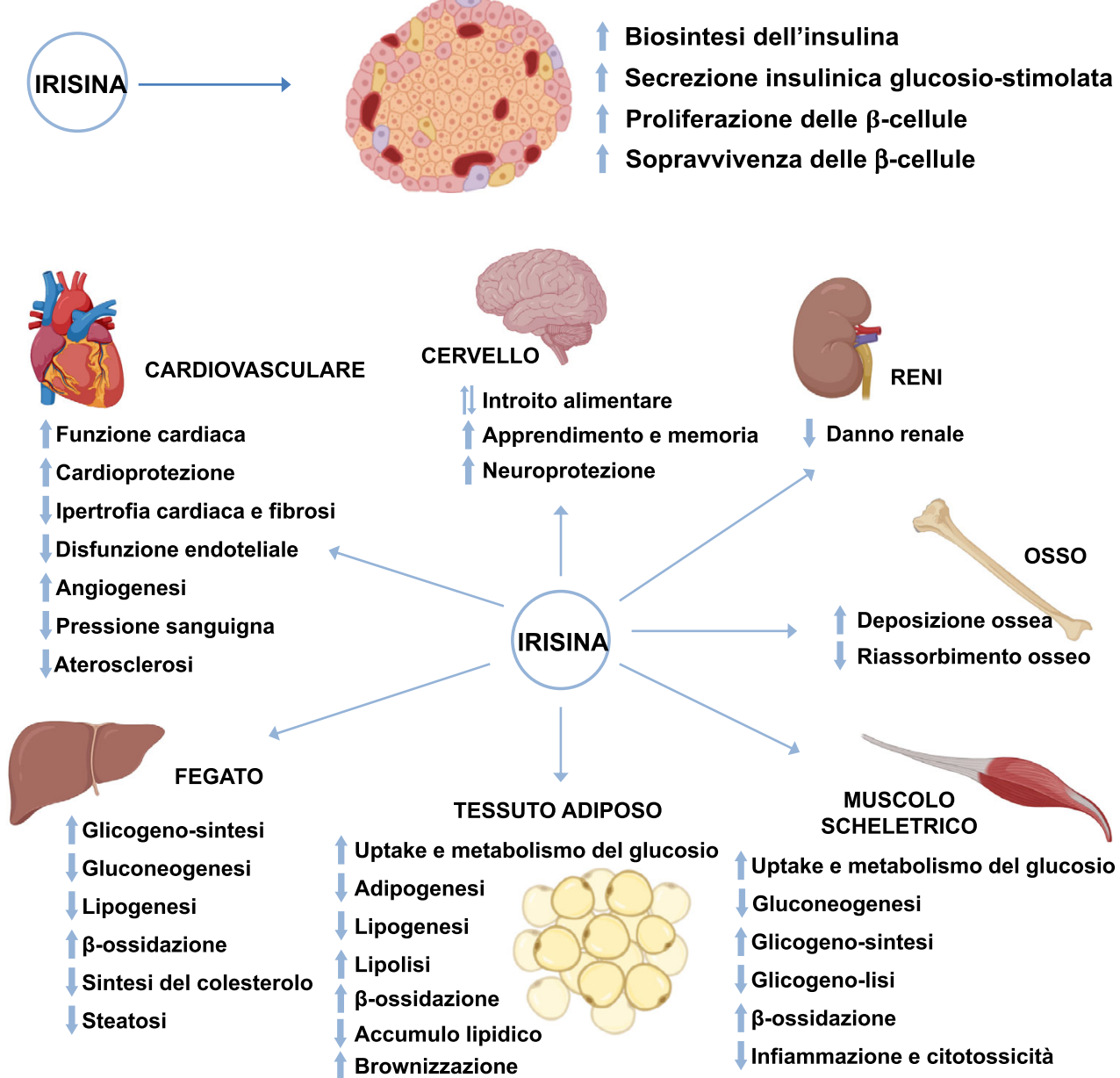

Numerosi studi hanno documentato la capacità dell'irisina di agire su muscolo scheletrico, tessuto adiposo e fegato, migliorando la sensibilità all'insulina, l'uptake del glucosio, riducendo la gluconeogenesi, la glicogenolisi e l'accumulo lipidico, e incrementando lo storage del glicogeno, la lipolisi e l'ossidazione degli acidi grassi [7]. Complessivamente, l'irisina esercita un'azione ipoglicemizzante e di riduzione degli accumuli lipidici (Fig. 2).

Da questi dati emerge come l'irisina sia in grado di migliorare sia la massa e la funzione delle beta-cellule pancreatiche, sia la sensibilità all'insulina dei tessuti periferici. Considerando che la ridotta massa-funzionale beta-cellulare e l'incrementata insulino-resistenza rappresentano i principali meccanismi patogenetici che portano all'insorgenza del DMT2, l'irisina si candida a diventare una nuova promettente molecola ad azione anti-diabetica. Tuttavia, ai nuovi farmaci anti-diabete, oltre a una spiccata azione ipoglicemizzante, viene richiesta anche la capacità di indurre una perdita di peso e di esercitare un'azione protettiva a livello cardio-renale. Diverse evidenze nei modelli animali mostrano che la somministrazione di irisina induce una significativa perdita di peso [7]. Tuttavia, non è ancora del tutto chiaro se l'irisina sia in grado di regolare il food intake mediante un'azione diretta sui centri ipotalamici deputati al controllo del senso di fame e sazietà. I dati a disposizione sono limitati e contrastanti e, pertanto, necessitano di ulteriori approfondimenti [7]. Alcuni lavori hanno infatti dimostrato la capacità dell'irisina di indurre l'espressione dei geni ipotalamici anoressizzanti cocaine- and amphetamine-regulated transcript (CART) e pro-opiomelanocortin (POMC) [10]; al contrario, in altri studi, l'irisina ha incrementato l'espressione di tali geni, suggerendo che gli effetti della somministrazione di irisina sul food intake possano dipendere dal timing e/o dalla via di somministrazione (Fig. 2) [7].

Studi recenti hanno dimostrato che l'irisina è in grado di proteggere il cuore dal danno indotto da ischemia/riperfusione, migliorare la funzione cardiaca e ridurre la dimensione delle aree infartuate, in modelli animali sia diabetici che non [7]. Inoltre, l'irisina migliora la performance cardiaca (aumentata frazione di eiezione e ridotta fibrosi) in topi $d b / d b$, normalmente caratterizzati da ridotta funzione cardiaca [7]. In aggiunta, l'irisina favorisce la vasodilatazione endoteliodipendente e, quindi, riduce la pressione sanguigna in topi 
obesi e in ratti che sviluppano spontaneamente ipertensione [7]. La riduzione della pressione sanguigna indotta dall'irisina è dovuta a diversi meccanismi, tra cui l'induzione del rilassamento delle arterie mesenteriche, l'attivazione dei neuroni che controllano il tono vagale cardiaco e l'aumento della vasodilatazione mediata dall'acetilcolina [7]. Infine, l'irisina è in grado di inibire l'aterosclerosi in topi carenti di apolipoproteina E, attraverso la riduzione dell'infiammazione vascolare indotta dalle LDL ossidate e induzione della proliferazione delle cellule vascolari [7]. Complessivamente, questi dati mostrano che l'irisina esercita una spiccata azione protettiva a livello cardiovascolare (Fig. 2).

In aggiunta, è stato dimostrato che l'irisina è in grado di ridurre il danno e la fibrosi renale, in diversi modelli animali e in linee cellulari, esitando in un miglioramento della funzione d'organo [11]. Nell'uomo, i livelli di irisina sono ridotti nei pazienti con malattia renale cronica [12], mentre sono significativamente e positivamente associati ai livelli di eGFR (Fig. 2) [13].

Infine, l'irisina ha mostrato una spiccata azione anabolica sull'osso, promuovendo la differenziazione degli osteoblasti, incrementando la massa corticale ossea e rendendo l'osso più resistente e meno suscettibile alle fratture (Fig. 2) [7]. Questi dati sono di particolare importanza per i pazienti affetti da diabete, caratterizzati da un maggiore rischio di fratture rispetto ai soggetti sani. A conferma di ciò, i livelli di irisina sono risultati essere inversamente correlati all'incidenza di fratture nelle donne in post-menopausa con osteoporosi e nei pazienti con DMT2 e patologie cardiovascolari (Fig. 2) [7].

\section{Livelli di irisina in corso di obesità e diabete mellito di tipo 2}

Come già accennato, i livelli di irisina sono più elevati nei soggetti obesi e positivamente correlati al Body Mass Index (BMI), indipendentemente dalla presenza o meno di alterazioni glicemiche [3]. È probabile che tale incremento rifletta una condizione di resistenza all'irisina o un aumento compensatorio dovuto alle iniziali anomalie metaboliche e all'iniziale stato di insulino-resistenza che sono caratteristici di questi pazienti [3-5]. Coerentemente, è stato dimostrato che una dieta ricca di grassi causa un rapido e persistente incremento delle concentrazioni sieriche di irisina, in topi sani [8]. In particolare, il rilascio di irisina sembrerebbe essere influenzato dal tipo di acidi grassi presenti nella dieta: esso è infatti favorito dagli acidi grassi saturi, più propensi a indurre insulino-resistenza, ma non dagli acidi grassi mono-insaturi, meno inclini a provocare danni metabolici e resistenza all'insulina [7, 8]. Inoltre, l'espressione genica di FNDC5 è incrementata nel muscolo scheletrico e nel tessuto adiposo di topi nutriti con una dieta ricca di grassi [14] e in miotubi umani trattati per brevi tempi con l'acido palmitico, principale rappresentante della categoria degli acidi grassi saturi [8].

La correlazione tra irisina e peso corporeo è confermata dal fatto che i livelli di irisina risultano significativamente ridotti in seguito a perdita di peso, mentre questa riduzione risulta vanificata nei pazienti che riacquistano il proprio peso iniziale [15]. Analogamente, i pazienti con anoressia nervosa hanno livelli di irisina circolanti inferiori del $15 \%$ rispetto ai soggetti di peso normale e del $30 \%$ rispetto agli individui obesi [16]. Inoltre, alcuni studi hanno riportato l'esistenza di una correlazione positiva tra i livelli sierici di irisina e i markers di adiposità e insulino-resistenza (massa grassa, circonferenza vita, rapporto vita-fianchi, indice HOMA-IR) e tra i livelli sierici di irisina e i disturbi dell'omeostasi glicemica e lipidica [7].

$\mathrm{Al}$ contrario, numerosi studi, comprese tre diverse metaanalisi, hanno mostrato una diminuzione delle concentrazioni di irisina in pazienti con DMT2, indipendentemente dalla durata di malattia e dalla presenza di terapia farmacologica in atto, e una concentrazione ancora più bassa in presenza di complicanze legate al diabete $[2,17,18]$. Tuttavia, ad oggi, non è ancora noto quali siano i meccanismi alla base dello switch tra gli alti valori di irisina circolanti nell'obesità e i bassi valori associati al DMT2. Alcuni studi in vitro hanno mostrato una riduzione dell'espressione genica di FNDC5 nei miotubi umani trattati in cronico con alte dosi di acidi grassi saturi o glucosio $[8,19]$. Questo suggerirebbe che la riduzione dei livelli di irisina in corso di diabete sia dovuta al protrarsi di un ambiente metabolicamente sfavorevole che, danneggiando direttamente il muscolo scheletrico, lo rende incapace di adeguare i livelli di secrezione di irisina in risposta al crescente scompenso metabolico sistemico.

Infine, i livelli di irisina sembrerebbero essere ulteriormente ridotti in presenza di complicanze micro e macrovascolari del diabete, per esempio in presenza di nefropatia e retinopatia diabetica e malattia coronarica, proponendo l'irisina come possibile marcatore di complicanze vascolari nei pazienti affetti da DMT2 [20].

\section{Conclusioni}

Fin dalla sua scoperta, l'irisina ha attirato l'attenzione della comunità scientifica per la sua capacità di favorire il browning del tessuto adiposo bianco, la termogenesi e il dispendio energetico, di ridurre il peso corporeo e di migliorare il metabolismo glucidico e lipidico. È importante sottolineare che i livelli circolanti di irisina sono ridotti nei pazienti affetti da DMT2, mentre sono più elevati in altri stati dismetabolici caratterizzati da insulino-resistenza (obesità, sindrome metabolica, sindrome dell'ovaio policistico), suggerendo la possibilità che l'alterazione dei livelli sierici di irisina possa contribuire ad alterare l'omeostasi metabolica ed energetica e promuovere l'insorgenza di dismetabolismi. 
Queste caratteristiche hanno reso l'irisina un candidato promettente per il trattamento di varie malattie metaboliche, in particolar modo il DMT2. L'irisina, infatti, possiede tutte le caratteristiche che vengono richieste a un farmaco antidiabete ideale: azione ipoglicemizzante glucosio-dipendente (rischio di ipoglicemie inesistente), capacità di ripristinare la massa funzionale beta-cellulare e di indurre perdita di peso, azione protettiva a livello cardio-renale. Tuttavia, ad oggi, l'irisina non è mai stata somministrata all'uomo; pertanto, non si hanno informazioni sulla sua efficacia, tollerabilità, farmacocinetica e farmacodinamica. Inoltre, sebbene il recettore dell'irisina sia stato identificato nel tessuto adiposo e nell'osso, questa scoperta dovrebbe essere confermata anche negli altri tessuti, tra cui le beta-cellule pancreatiche. Infatti, i modelli animali knock-out tessuto-specifico per il recettore dell'irisina ci aiuterebbero a ottenere un quadro più chiaro dell'importanza degli effetti metabolici dell'irisina in vivo. Conoscere il recettore dell'irisina ci aiuterebbe anche a ingegnerizzare la sua struttura proteica per migliorarne l'affinità di legame al proprio recettore e, pertanto, l'efficacia. Tali molecole derivanti dalla razionalizzazione della struttura dell'irisina potrebbero dare origine a una nuova classe farmacologica per il trattamento del diabete: gli "analoghi dell'irisina" o gli "agonisti del recettore dell'irisina”.

Informazioni Supplementari La versione online contiene materiale supplementare disponibile su https://doi.org/10.1007/s40619-02201046-z.

Funding Note Open access funding provided by Università degli Studi di Bari Aldo Moro within the CRUI-CARE Agreement.

\section{Dichiarazioni etiche}

Conflitto di interesse Gli autori Francesco Giorgino, Annalisa Natalicchio e Nicola Marrano sono inventori di un brevetto dal titolo Pharmacological use of a myokine able to preserve the function and mass of the pancreatic cells under dysmetabolic conditions.

Consenso informato Lo studio presentato in questo articolo non ha richiesto sperimentazione umana.

Studi sugli animali Gli autori di questo articolo non hanno eseguito studi sugli animali.

Open Access This article is licensed under a Creative Commons Attribution 4.0 International License, which permits use, sharing, adaptation, distribution and reproduction in any medium or format, as long as you give appropriate credit to the original author(s) and the source, provide a link to the Creative Commons licence, and indicate if changes were made. The images or other third party material in this article are included in the article's Creative Commons licence, unless indicated otherwise in a credit line to the material. If material is not included in the article's Creative Commons licence and your intended use is not permitted by statutory regulation or exceeds the permitted use, you will need to obtain permission directly from the copyright holder. To view a copy of this licence, visit http://creativecommons.org/licenses/by/4. $0 \%$

\section{Bibliografia}

1. Boström P, Wu J, Jedrychowski MP et al (2012) A PGC1- $\alpha$ dependent myokine that drives Brown-fat-like development of white fat and thermogenesis. Nature 481:463-468

2. Song R, Zhao X, Zhang D-Q et al (2021) Lower levels of irisin in patients with type 2 diabetes mellitus: a meta-analysis. Diabetes Res Clin Pract 175:108788

3. Qiu S, Cai X, Yin H et al (2016) Association between circulating irisin and insulin resistance in non-diabetic adults: a meta-analysis. Metabolism 65:825-834

4. Arhire LI, Mihalache L, Covasa M (2019) Irisin: a hope in understanding and managing obesity and metabolic syndrome. Front Endocrinol (Lausanne) 10:524

5. Cao RY, Zheng HC, Damian R, Yang J (2019) FNDC5: a novel player in metabolism and metabolic syndrome. Biochimie 158:111-116

6. Wang C, Zhang X-Y, Sun Y et al (2018) Higher circulating irisin levels in patients with polycystic ovary syndrome: a meta-analysis. Gynecol Endocrinol 34:290-293

7. Marrano N, Biondi G, Borrelli A et al (2021) Irisin and incretin hormones: similarities, differences, and implications in type 2 diabetes and obesity. Biomolecules 11:286

8. Natalicchio A, Marrano N, Biondi G et al (2017) The myokine irisin is released in response to saturated fatty acids and promotes pancreatic $\beta$-cell survival and insulin secretion. Diabetes 66(11):2849-2856

9. Marrano N, Natalicchio A, Biondi G et al (2020) 2094-P: effects of irisin on human pancreatic islets from type 2 diabetic subjects. Diabetes 69(Suppl 1):294-P

10. Natalicchio A, Marrano N, Biondi G et al (2019) Irisin increases the expression of anorexigenic and neurotrophic genes in mouse brain. Diabetes/metab Res Rev 36(3):e3238

11. Liu Y, Fu Y, Liu Z et al (2020) Irisin is induced in renal ischemiareperfusion to protect against tubular cell injury via suppressing p53. Biochim Biophys Acta, Mol Basis Dis 1866(7):165792

12. Wen MS, Wang CY, Lin SL, Hung KC (2013) Decrease in irisin in patients with chronic kidney disease PLoS One 8(5):e64025

13. Ebert T, Focke D, Petroff D et al (2014) Serum levels of the myokine irisin in relation to metabolic and renal function. Eur $\mathbf{J}$ Endocrinol 170:501-506

14. Guilford BL, Parson JC, Grote CW et al (2017) Increased FNDC5 is associated with insulin resistance in high fat-fed mice. Physiol Rep 15(13):e13319

15. Crujeiras AB, Pardo M, Roca-Rivada A et al (2014) Longitudinal variation of circulating irisin after an energy restriction-induced weight loss and following weight regain in obese men and women. Am J Human Biol 26:198-207

16. Stengel A, Hofmann T, Goebel-Stengel M et al (2013) Circulating levels of irisin in patients with anorexia nervosa and different stages of obesity - correlation with body mass index. Peptides 39:125-130

17. Zhang C, Ding Z, Lv G et al (2016) Lower irisin level in patients with type 2 diabetes mellitus: a case-control study and meta-analysis. J Diabetes 8:56-62

18. Du XL, Jiang W-X, Lv Z-T (2016) Lower circulating irisin level in patients with diabetes mellitus: a systematic review and metaanalysis. Horm Metab Res 48:644-652

19. Kurdiova T, Balaz M, Vician M et al (2014) Effects of obesity, diabetes and exercise on Fndc5 gene expression and irisin release in human skeletal muscle and adipose tissue: in vivo and in vitro studies. J Physiol 592:1091-1107

20. Polyzos SA, Anastasilakis AD, Efstathiadou ZA et al (2018) Irisin in metabolic diseases. Endocrine 59:260-274

Nota della casa editrice Springer Nature rimane neutrale in riguardo alle rivendicazioni giurisdizionali nelle mappe pubblicate e nelle affiliazioni istituzionali. 